\title{
Dynamic Augmented Reality for Sensory Substitution in Robot-Assisted Surgical Systems
}

\author{
Takintope Akinbiyi ${ }^{1}$, Carol E. Reiley ${ }^{1}$, Sunipa Saha ${ }^{1}$, Darius Burschka ${ }^{2}$, \\ Christopher J. Hasser ${ }^{3}$, and David D. Yuh ${ }^{4}$, and Allison M. Okamura ${ }^{1}$
}

\begin{abstract}
Teleoperated robot-assisted surgical systems provide surgeons with improved precision, dexterity, and visualization over traditional minimally invasive surgery. The addition of haptic (force and/or tactile) feedback has been proposed as a way to further enhance the performance of these systems. However, due to limitations in sensing and control technologies, implementing direct haptic feedback to the surgeon's hands remains impractical for clinical application. A new, intuitive augmented reality system for presentation of force information through sensory substitution has been developed and evaluated. The augmented reality system consists of force-sensing robotic instruments, a kinematic tool tracker, and a graphic display that overlays a visual representation of force levels on top of the moving instrument tips. The system is integrated with the da Vinci Surgical System (Intuitive Surgical, Inc.) and tested by several users in a phantom knot tying task. The augmented reality system decreases the number of broken sutures, decreases the number of loose knots, and results in more consistent application of forces.
\end{abstract}

\section{INTRODUCTION}

Sensory deprivation in endoscopic procedures presents a significant limitation for the surgeon. Thus, teleoperated surgical systems have been designed to provide improvements over traditional minimally invasive surgery (MIS) in precision, dexterity, and visualization. Such systems have been used worldwide in thousands of procedures [1], [2]. One successful system, which is used in our work, is the da Vinci Surgical System (Intuitive Surgical, Inc., Sunnyvale, CA) [3]. Although this teleoperated system has the capability of providing haptic feedback, the level of haptic feedback to the operator is set so low for performance reasons that the effect of haptic information during delicate surgical maneuvers is negligible. It has been shown both anecdotally [2] and experimentally [4] that the lack of haptic feedback affects the performance of several surgical tasks. In this study, we propose a method of sensory substitution (SS) that provides an intuitive form of haptic feedback to the user. We evaluate human performance of a surgical knot tying task (Figure 1) involving precise hand maneuvers with and without this method of indirect haptic feedback.

\footnotetext{
${ }^{1}$ Engineering Research Center for Computer Integrated Surgical Systems and Technology, Johns Hopkins University, Baltimore, MD 21218. Email: \{tope,creiley,ssaha,aokamura\}@jhu.edu

${ }^{2}$ Department of Computer Science, Technische Universitat Munchen, Munich, Germany. Email: burschka@cs.tum.edu

${ }^{3}$ Intuitive Surgical, Inc., Sunnyvale, CA 94086. Email: chris.hasser@intusurg.com

${ }^{4}$ Johns Hopkins Medical Institutions, Division of Cardiac Surgery, Baltimore, MD 21287-4618. Email: dyuh@csurg.jhmi.jhu.edu
}
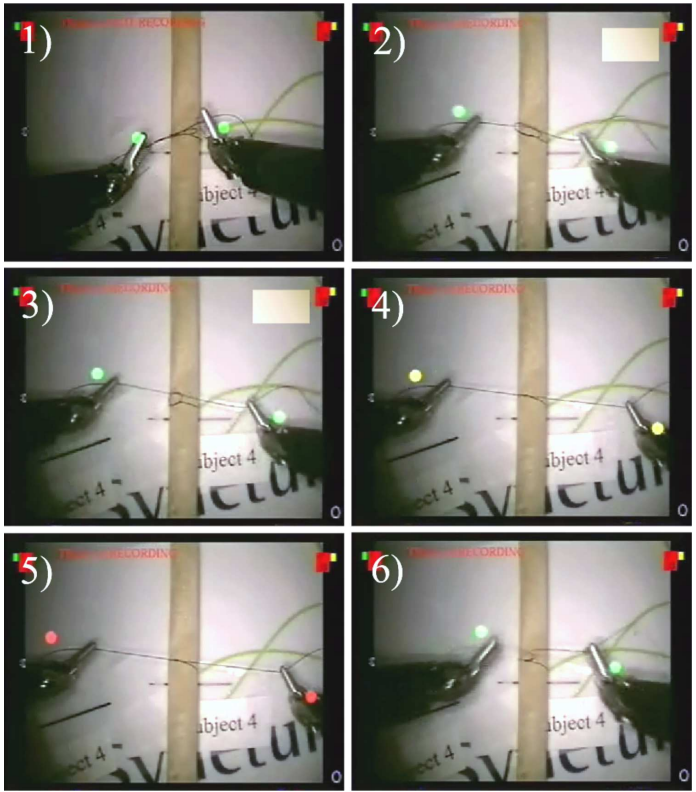

Fig. 1. Sequential snapshots of a knot tying task. The colors of the circles overlaid on the endoscopic images provide information about applied force

Research has shown that haptic feedback can improve operator performance. During a blunt dissection, Wagner et al. [5] found that the presence of force feedback in a teleoperated system reduced the peak force magnitude by a factor of two and the number of errors by a factor of three. Moreover, they show that increased feedback gains resulted in better user performance. However, force feedback is difficult to relay directly to the surgeon's hands because of sensing and control limitations. For natural haptic interaction, the force/torque sensing should have the same number of degrees of freedom (DOF) as the DOF of motion of the system. In robot-assisted surgical systems, which typically have 7 DOF per arm, it is challenging to create a force/torque sensing method with the sufficient DOF and appropriate size, biocompatibility, sterilizability, and cost (for disposable tools). Although user performance may not be significantly affected by using fewer DOF of force/torque feedback [6], this can lead to non-passive and potentially unstable system behavior [7]. Thus, practical force-sensing schemes with fewer DOF require the exploration of alternative methods for haptic feedback.

As direct force feedback may not always be possible to implement, recent work has focused on the use of SS to relay 


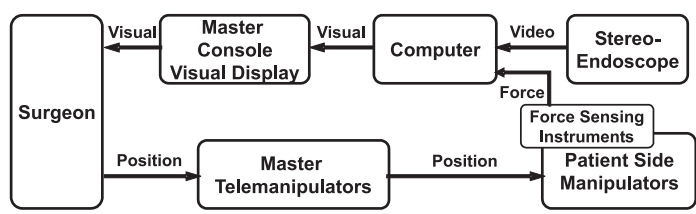

Fig. 2. System diagram of the sensory substitution system.

haptic information to the surgeon. Studies on non-surgical teleoperation systems show that SS of haptic information can enhance the ability of the operator to sense the environment and control the robot ([8], [9]). Sabatini, et al. presented force information from a strain gage sensor visually to users for a microsurgical task [12]. This approach was validated for minimally invasive robot-assisted surgery by Kitagawa, et al., who implemented a simple overlay to aid users performing a knot tying task with the da Vinci system [10]. They found that the coefficient of variance for robotic ties with SS of force information was lower than that of hand ties, demonstrating the potential for simple SS in robot-assisted MIS. Tavakoli, et al. used a visual display to display changes in applied force [11]. They found that visual information can reduce peak and average forces as compared with pure haptic feedback. The prior work uses static windows locked at the corner of the endoscopic image, while our work provides an intuitive overlay that tracks and moves with the tool in real time. Our work is also the first to provide SS using force sensors directly on the tools of a clinical surgical system.

\section{Augmented Reality System Design}

The augmented reality system presented in this paper is inexpensive and can be integrated into existing robot-assisted surgical systems. Figure 2 shows how the force and video components are integrated into the da Vinci. For our knottying experiment described in Section III, users were asked to tighten a loose knot. During the experiments, the video footage was displayed in real time to the user through the $3 \mathrm{D}$ console and to the investigators on a TV screen. Both video and forces applied to sutures by da Vinci instruments were recorded for analysis purposes. The user applies a force to the manipulated tissues and this force is graphically represented and overlaid on the streaming video from the camera. The visual overlay for force consists of two circles that track the location of its corresponding instrument as they move freely in space and visually display the amount of force sensed on a surgical instrument controlled by the da Vinci Surgical System. An expert da Vinci surgeon involved in the experimental design set the thresholds for the circles to change colors by completing the task and noting the force at significant moments. These overlays are semitransparent circles that discretely change color across three pre-determined ranges according to the amount of bending forces detected by the strain gages:

1) Low Force Zone (LFZ): (green color) Represents forces that are observed when low or no force is applied.

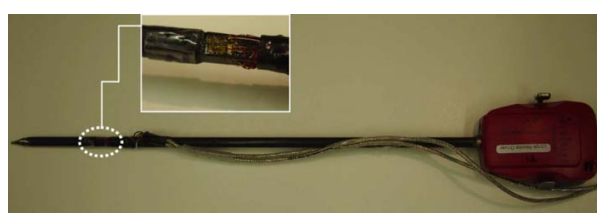

Fig. 3. Modified da Vinci instruments with strain gages in a Wheatstone full bridge configuration. Inset picture: Close-up of the strain gage and wiring.

2) Ideal Force Zone (IFZ): (yellow color) Represents forces that are observed if the tension of the suture reaches the desired range. Suture tied at this tension will not over-constrict underlying tissue but is still strong enough to prevent the knot from slipping along the rod. Novices typically have a difficult time knowing when the knot is secure and will continue pulling until the suture breaks.

3) Excessive Force Zone (EFZ): (red color) Represents forces that are observed when excessive force is used. This could be when a user attempts to pull a knot at an incorrect angle or when a subject pulls too hard on a knot that is already tight, resulting in a broken suture.

For this experiment, the two original instruments on the da Vinci were replaced by modified large needle drivers (Intuitive Surgical Inc.). Inexpensive strain gages are placed on the instrument's shaft in a full Wheatstone bridge arrangement to measure the forces being applied at each instrument's tip (Figure 3). No forces other than those sensed by the modified tools are displayed to the subject.

\section{EXPERIMENT PROCEDURE}

Using the da Vinci Surgical System, subjects were asked to tighten the surgeon's knots around a flexible rubber tube. The loose surgeon's knot was located at the center of the rubber rod with the first throw tight against the rod and the second throw loose. The ties were performed with a 5-0 silk suture, $12 \mathrm{~cm}$ in length. Both ends of the unfinished knot were visible in the da Vinci endoscopic images. The task was isolated to the final step of securing the knot since that was the most frequent step in the knot tying procedure where excessive force causes permanent damage to the vessel. Subjects were instructed to pull outwards in a direction perpendicular to the rod (Figure 4).

After ten minutes of practice time, each subject had ten

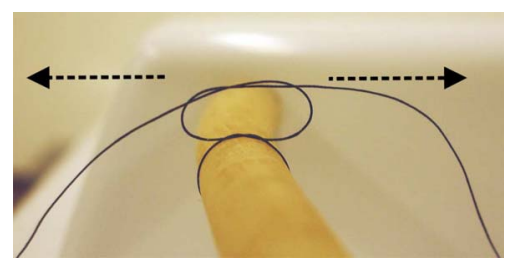

Fig. 4. Initial state of surgeon's knot on a knot tying board at start of trial. The arrows show the direction which subjects were instructed to pull the ends of the suture. Note that the first throw is tight against the rod and the second throw is loose. 
TABLE I

NUMBER OF SUTURES BROKEN WITH AND WITHOUT SS

\begin{tabular}{|l||c|c|c|c|c|c|c|c|c|c|}
\hline \multicolumn{1}{|c||}{ Overlay } & \multicolumn{10}{c|}{ Subject ID } \\
\hline \hline & 1 & 2 & 3 & 4 & 5 & 6 & 7 & 8 & 9 & Total \\
\hline Off & 3 & 2 & 1 & 1 & 0 & 0 & 1 & 4 & 2 & 14 \\
\hline On & 0 & 0 & 0 & 0 & 0 & 0 & 0 & 0 & 0 & 0 \\
\hline
\end{tabular}

trials with the overlay on and ten with the overlay off in a random order. For each subject, the first trial with the overlay on and the first trial with the overlay off were thrown out during data analysis to ensure that subjects were familiar with the task procedure. Therefore, twenty trials were run while only eighteen trials were used for data analysis.

The experiment consisted of nine subjects. All subjects, one surgeon and eight non-surgeons, considered themselves novice da Vinci users. The gender of the subjects consisted of six males and three females, all right handed with the average age being 25.8 years old. The surgeon was included as a control with which we were able to compare and quantify the performance of the eight novice users, both with and without SS.

\section{RESUlTS AND DISCUSSION}

Using a significance level of $p=0.05$, we conducted a three-way mixed effects analysis of variance (ANOVA) with SS, subject, and overlay randomization group included as factors.

\section{A. Number of Broken Sutures}

The experimental data support the hypothesis that SS can reduce the number of sutures that break while completing a knot indicating improvement in performance. No sutures were broken with SS and 14 were broken without SS (Table I). Subjects are more equipped to consistently reach the maximum safe tension with SS, thereby eliminating the occurrence of broken sutures caused by overextension.

\section{B. Precision:}

This is a binary metric of whether the knot was considered ideal or loose based on the quality of the knot. In this study, SS decreased the number of knots considered loose by $27 \%$ (Table II). Four of the nine subjects had no loose knots with SS while only two of the subjects had no loose knots without SS. This result is significantly different with a p-value of 0.0667 . We believe that more testing and a larger experiment base may improve this metric. In many routine surgical tasks, such as knot tying, haptic feedback is

TABLE II

NUMBER OF LOOSE KNOTS TIED WITH AND WITHOUT SS

\begin{tabular}{|l||c|c|c|c|c|c|c|c|c|c|}
\hline \multicolumn{1}{|c||}{ Overlay } & \multicolumn{10}{|c|}{ Subject ID } \\
\hline \hline & 1 & 2 & 3 & 4 & 5 & 6 & 7 & 8 & 9 & Total \\
\hline Off & 3 & 2 & 6 & 0 & 2 & 3 & 0 & 1 & 1 & 18 \\
\hline On & 2 & 0 & 4 & 0 & 1 & 4 & 0 & 0 & 2 & 13 \\
\hline
\end{tabular}
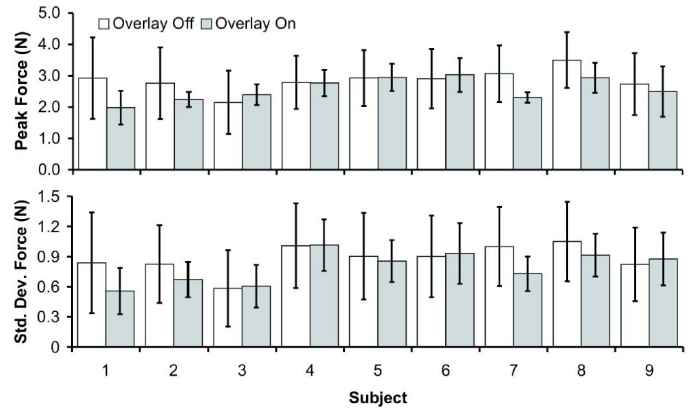

Fig. 5. Top: The peak applied force with and without overlay $(p=0.0539)$. Bottom: The standard deviation of applied force with and without overlay. $(\mathrm{p}=0.0414)$.

needed; especially when surgeons deal with delicate tissues like those encountered during cardiac surgery. If the suture is inserted correctly, over tightening the suture knot can cause permanent damage to the vessel. In such cases, haptic feedback can be used by the surgeon to determine how to pull the suture so that the knot securely holds tissue without damaging it.

\section{Peak and Standard Deviation of Applied Force:}

The peak applied force was the maximum amount of force exerted by the subject throughout the course of each trial by either the right or left instruments of the da Vinci. The standard deviation was calculated as the standard deviation of both the right and left instrument forces from the mean force for each trial. The application of lowered peak forces is desirable because underlying tissue is subject to less damaging stresses while decreased standard deviations results in less variance in applied force and hence more consistency in user performance. Thus, a decrease in the peak applied force and standard deviation of force, providing that the knot remains tied, is seen as an improvement in performance.

SS was not able to significantly improve the peak applied force metric (Figure 5) as shown by the p-value of 0.0539 . This $\mathrm{p}$-value indicates that more sensitive tests involving a larger number of subjects are needed to sufficiently determine the significance. The hypothesis that SS can decrease the standard deviation of applied forces (Figure 5) is supported by the experimental data with a p-value of 0.0414. SS was able to help subjects repeatedly apply the desired magnitude of force.

\section{Task Completion Time}

Task completion time is a measure of the total time it took the subject to complete the given task starting from the time he/she first gripped the suture and began pulling to the time he/she released the suture. A decrease in this metric is seen as an improvement in performance. The presence of SS was not able to improve the subject's performance with respect to this metric $(p=0.7931)$ because there was significant variation between times for different subjects (Figure 6). From observations made during the experiment, it is clear that each subject approached the task in drastically 
TABLE III

SUMMARY OF ANOVA RESULTS

\begin{tabular}{|l||c|c|}
\hline Metric & p-value & significant \\
\hline \hline Num. of broken sutures & 0.0111 & $\mathrm{Y}$ \\
\hline Precision & 0.0667 & $*$ \\
\hline Peak Applied Force & 0.0539 & $*$ \\
\hline Standard Deviation of Applied Force & 0.0414 & $\mathrm{Y}$ \\
\hline Time & 0.7934 & $\mathrm{~N}$ \\
\hline \multicolumn{2}{|c|}{ *p-value slightly below the significance level $(\mathrm{p}=0.05)$}
\end{tabular}

different ways. Some used the SS as was suggested by the experimenters and stopped tightening the knot once the IFZ had been passed for the first time. Others maintained forces past IFZ for a period of time in order to ensure a tight knot.

\section{E. Summary}

Table III lists a summary of the results of the ANOVA analysis. Besides the quantitative data presented above, there are many qualitative advantages of our system. In a feedback survey, $100 \%$ of the subjects preferred the overlay on and trusted the threshold values that were set. One user added that SS helped much more than he thought it would. Many used it as a way to determine how much force/tension to apply. The overlay gave them "a sense of confidence" in completing the task and when available, they used it "all the time." This additional confidence that the presence of force information instills on subjects can be very advantageous in the training of medical personnel, especially those who are unfamiliar with robot-assisted surgical systems.

All subjects felt that completing the task was much easier with the overlay on. Without the overlay, subjects relied solely on visual cues such as the deformation of the rubber tube, which means that in a clinical application, a surgeon would have damaged tissue already. For tasks without the element of elasticity or in cases where the view is slightly obstructed, considerable damage to the tissue might result.

\section{CONClusions And Future Work}

The dynamic augmented reality system presented here can aid surgeons in tying secure knots and reduce broken sutures. It is more flexible and intuitive to use than previous sensory substitution systems, and was fully integrated with a commercially available robot-assisted surgical system. In our study, users achieved greater consistency in both the mean and peak applied forces with sensory substitution as indicated by smaller standard deviations.

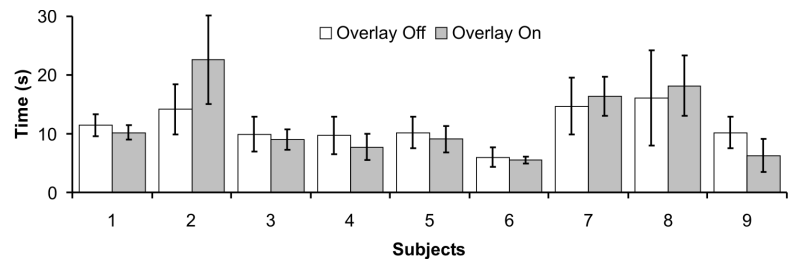

Fig. 6. Mean task completion time with and without overlay. $(p=0.7931)$.
Future research efforts will focus on identifying the potential of sensory substitution to improve performance among different groups, including surgeons versus non-surgeons and da Vinci experts versus non-experts. The next step will then be to examine the role of force feedback with various size sutures, environments of varying stiffness, and different surgical tasks. Our ultimate goal is to use the augmented reality system clinically to help in surgeon training and reduce errors in robot-assisted surgical procedures.

\section{ACKNOWLEDGMENT}

This work was supported in part by NSF (EEC9731478), Whitaker Foundation (RG-02-911), and NIH (R01-EB002004). The authors are grateful to Dr. Randy Brown, Sue Eller, and the staff of Minimally Invasive Surgical Training Center at Johns Hopkins Medical Institutions for access to the da Vinci Surgical System, Intuitive Surgical, Inc. for providing an Application Programmer's Interface, and Dr. Russell Taylor, Henry Lin, and Lawton Verner for technical advice.

\section{REFERENCES}

[1] E. A. Grossi, et al., "Case Report of a Robotic Instrument-Enhanced Mitral Valve Surgery," The Journal of Thoracic and Cardiovascular Surgery, 120(6):1169-1171, 2000.

[2] F. W. Mohr, et al., "Computer-Enhanced "Robotic" Cardiac Surgery: Experience In 148 Patients," The Journal of Thoracic and Cardiovascular Surgery, 121(5):842-853, 2001.

[3] G. Guthart and J. K. Salisbury, "The Intuitive $\mathrm{e}^{T M}$ Telesurgery System: Overview and Application," IEEE International Conference On Robotics and Automation, pp. 618-621, 2000.

[4] B. T. Bethea, et al. "Application of Haptic Feedback To Robotic Surgery," Journal of Laparoendoscopic and Advanced Surgical Techniques, 14(3):191-195, 2004.

[5] C. R. Wagner, et al., "The Role of Force Feedback In Surgery: Analysis of Blunt Dissection," In 10th Symposium On Haptic Interfaces For Virtual Environments and Teleoperator Systems, pp. 68-74, 2002.

[6] W. Semere, et al., "Teleoperation with Sensor/Actuator Assymetry: Task Performance with Partial Force Feedback," Proceedings of the 12th Symposium on Haptic Interfaces or Virtual Environment and Teleoperator Systems, pp. 121-127, 2004

[7] L. N. Verner and A. M. Okamura, "Sensor/Actuator Asymmetries in Telemanipulators: Implications of Partial Force Feedback," 14th Symposium on Haptic Interfaces for Virtual Environments and Teleoperator Systems, pp. 309-314, 2006.

[8] M. J. Massimino and T. B. Sheridan, "Sensory Substitution For Force Feedback In Teleoperation," Presence: Teleoperators and Virtual Environments, 2(4):344-352, 1993.

[9] P. Richard and P. Coiffet, "Human Perceptual Issues In Virtual Environments: Sensory Substitution and Information Redundancy," Robot and Human Communication Proceedings of the IEEE International Workshop, pp. 301-306, 1995.

[10] M. Kitagawa, et al. "Effect of Sensory Substitution On Suture Manipulation Forces For Robotic Surgical Systems," Journal of Thoracic and Cardiovascular Surgery, 129(1):151-158, 2005.

[11] M. Tavakoli, et al. "Robotic Suturing Forces in the Presence of Haptic Feedback and Sensory Substitution," Proceedings of the 2005 IEEE Conference on Control Applications, pp. 1-6, 2005.

[12] A. M. Sabatini, et al., "Force Feedback Based Telemicromanipulation For Robot Surgery On Soft Tissues," Proceedings of the IEEE Engineering in Medicine and Biology Society 11th Annual International Conference, pp. 890-891, 1989. 